\title{
In Memory of Aleksandr Borisovich Devin (1944-2007)
}

\section{DOI: $10.1134 / \mathrm{S} 1022795408120132$}

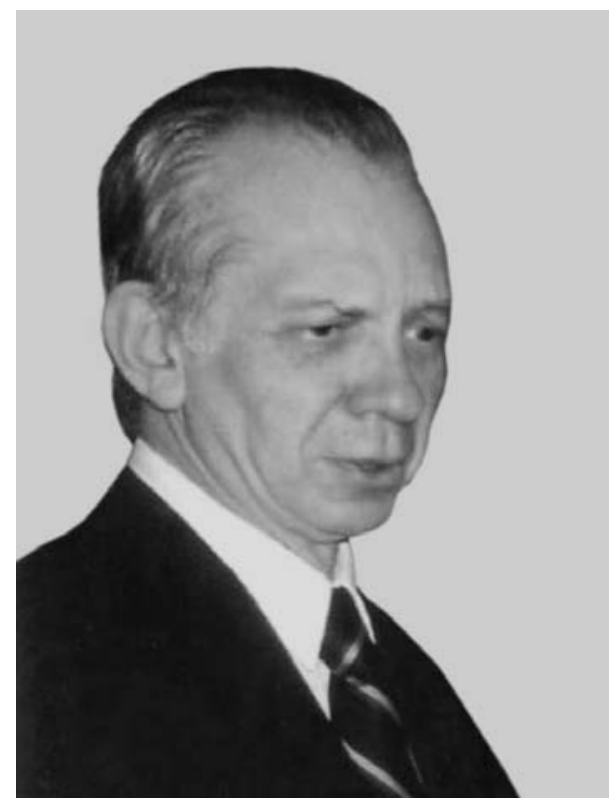

On June 20, 2007, passed away Aleksandr Borisovich Devin, a renown geneticist, doctor of biological sciences. He was an outstanding person, known for his erudition, scrupulous approach to any problem, and devotion to science. Aleksandr Devin worked at the Institute of Molecular Genetics, Russian Academy of Sciences (Moscow), his favorite object was Sacchromycetes yeasts, and his area of research, genetic control of stability of hereditary structures.

In his early works on the role of repair systems in cell genetic processes, Devin demonstrated that hypothesis of $\mathrm{Ch}$. Auerbach on different mechanisms of appearance of complete and mosaic mutations was inconsistent (Arman, Devin, 1969). Thorough genetic investigations, conducted by Devin together with
O.N. Kurennaya (Vavilov Institute of General Genetics, Russian Academy of Sciences) allowed refining the mechanism of "replicating instability" (Kurennaya, Devin, 1985). He has discovered interclonal variability of cell susceptibility to lethal and mutagenic action of nitrous acid and revealed the unstable factor (mitochondrial genome), causing this instability (Devin, Koltovaya, 1983).

A priority result of Aleksandr Devin studies is also the detection of genetic determination of low stability of Saccharomycetes mitochondrial genome (Devin, Koltovaya, 1981). The isolated genes, including identified genes CDC28, NET1, and HFI1, occupy a high position in the hierarchy of general genetic regulation in Saccharomyces cerevisiae and coordinate stability of both mitochondrial and nuclear genomes (Devin, Koltovaya, 1981; Koltovaya et al., 2003). Further investigations have shown that a decrease in genetic stability, as well as an increase in susceptibility of the mutant cells to inactivating action of ionizing radiation were partially explained by the involvement of these genes in checkpoint control (Koltovaya et al., 1998; Koltovaya et al., 1998, 2008). It is known that checkpoint control arrests the cell cycle in case of DNA damage, thus providing time for repair of the lesions. Yeast is a good model object to study this vitally important process, since a disturbance of the checkpoint control leads to tumor transformation of the cells. The importance of studies conducted by Alexandr Devin is also due to the fact that numerous diseases caused by mitochondrial genome impairments were revealed. Investigation of the role of distinct regulatory genes in determination of genetic stability, mechanism of control of the low stability level in the mitochondrial genome, and its role in cell life cycle have not been completed. They will be continued by young researchers, but friends and colleagues of Aleksandr Devin will miss his friendly concern and keen evaluation of the scientific results. 